\title{
4-Aminothiophenol Strong SERS Signal Enhance- ment at Electrodeposited Silver Surface
}

\author{
Lenka Škantárová ${ }^{*}, \quad$ Andrej Oriňák² ${ }^{2}$ Renáta Oriňákováa ${ }^{2}$, František Lofaj³
}

(Received 4 July 2012; accepted 21 September 2012; published online 25 September 2012.)

\begin{abstract}
Strong surface enhancement Raman spectroscopy signal of 4-aminothiophenol on silver substrate prepared by controlled electrodeposition is documented in this article. Enhancement factor was found to be affected not only by nanoparticle size, shape, orientation and spatial distribution, but also by interaction of Ag nanoparticles with thiol group of testing analyte. Self-assembled monolayers formation was contributed to this unique signal enhancement. The enhancement factor was established of $1.81 \times 10^{14}$.
\end{abstract}

Keywords: Silver; 4-Aminothiophenol; Surface-Enhanced Raman spectroscopy

Citation: Lenka Škantárová, Andrej Oriňák, Renáta Oriňáková and František Lofaj, "4-Aminothiophenol Strong SERS Signal Enhancement at Electrodeposited Silver Surface", Nano-Micro Lett. 4 (3), 184-188 (2012). http://dx.doi.org/10.3786/nml.v4i3.p184-188

\section{Introduction}

New insight into the fundamentals of electrodeposition can be provided by studying the initial nanoparticle formation at the beginning of electrocrystallization process. Surprising phenomena like band splitting observed when monitoring the surface-enhanced Raman spectroscopy (SERS) activity of the adsorbate molecule in the process of electrocrystallization, indicated some interaction between particle growth and SERS activity [1]. Electrochemical cycling of silver surfaces in the presence of the organophosphonates paraoxon and malathion leads to changes in the electrochemical response of silver and the formation of silver nanostructures. The size of the nanostructures greatly influences the SERS signal and the strongest enhancement is observed for mid-sized nanostructures with a uniform thickness on the surface. The limit of detection was shown to be in the range of $10 \mathrm{nM}$ to $10 \mathrm{pM}$ for paraoxon and malathion, respectively [2].

SERS technique has become widely used for identifying and providing structural information about molec- ular species in low concentration. There is an ongoing interest in finding optimum particle size, shape and spatial distribution for optimizing the SERS substrates and pushing the sensitivity toward the single-molecule detection limit [3]. Though the phenomenon of SERS is still under discussion, it is widely accepted that the origin of SERS is closely correlated to the enhancement of the local electromagnetic field at the surface of small metallic nanoparticles and of the charge transfer between adsorbates and the metal particles [4-6].

It has been shown that the oscillation of electrons at the metal dielectric interface is strongly dependent on the size, symmetry, and proximity of nanoparticles [7]. In addition, small metal particles of some metals ( $\mathrm{Ag}$, $\mathrm{Cu}, \mathrm{Au}$ ) have shown tremendous enhancement factors for Raman scattering, and thus enabling Raman spectroscopy of single molecules [8]. Presently, the surface enhancement effect of metals was explained as the result of multiple cooperating mechanisms $[4,5,9]$. Their role and the contributions have not yet been quantitatively clarified. There seems to be agreement that SERS is a function of the roughness features of the enhancing

\footnotetext{
${ }^{1}$ Department of Analytical Chemistry, Faculty of Sciences, Comenius University, Mlynská dolina, SK-842 15, Bratislava 4, Slovak Republic

${ }^{2}$ Department of Physical Chemistry, Faculty of Science, P. J. Šafárik University, Moyzesova 11, SK-041 54, Košice, Slovak Republic

${ }^{3}$ Institute of Materials Research, Slovak Academy of Sciences, Watsonova 47, SK-04353, Košice, Slovak Republic

*Corresponding author. E-mail: Skantarova.Lenka@gmail.com
} 
surface. Therefore, the preparation of SERS active particles with well defined size and morphology can lead to a better theoretical understanding of SERS, and thus enhancing the analytical importance of this method.

The maximum SERS enhancement factors found in the relevant measurements were $10^{10}$ for silver particles and $10^{8}$ for gold particles. The optical and spectroscopic data of the local nanoparticle structures investigated showed that SERS is a local phenomenon, because only few particles are spectral active. The strongest enhancements in SERS were reported from particle agglomerates, typically the Raman radiation is emitted from irregular structures like the necks between two or more particle agglomerated [10]. In an effort to achieve high SERS signal enhancement in porous alumina-membrane-based substrates optimization of pore diameter, the placement of nanoparticles, and the transmission of SERS substrates were investigated. The alumina-membrane-based substrates with a pore diameter of $355 \mathrm{~nm}$ incorporating silver nanoparticles put forward very high SERS activity with enhancement factors of $10^{10}$ [11].

The biocompatible SERS substrate based on small clusters of anisotropic silver nanoparticles embedded in a film of chitosan biopolymer is sensitive enough to be implemented as effective plasmonic substrate for SERS detection of nonresonant analytes at the single-molecule level. The SERS efficiency of the biocompatible film is assessed by employing Raman imaging and spectroscopy of adenine, a significant biological molecule [3]. The SERS signal was enabled by gold nanoparticles attached to MPO, pAb and their immunocomplex at an excitation wavelength of $785 \mathrm{~nm}$. Comparative SERS spectrum analysis of MPO, pAb, and their immunocomplex reveals the significant peak shifts and intensity variations caused by the conformational changes due to the immunocomplex formation. This demonstrates the capability of SERS to identify binding events and differentiate an immunocomplex from its unbound components with direct applications in biosensors [12]. The optimum size of spherical silver nanoparticles (AgNPs) for off-resonance SERS was found to be $\sim 50 \mathrm{~nm}$ based on the equivalent Ag content in AgNP colloids [13].

Mercaptoacetic acid-capped spherical silver nanoparticles with a diameter of about $17 \mathrm{~nm}$ were prepared by a simple chemical reaction. The results show that the spherical and rodlike particles were formed at the beginning of the reaction, and then the rodlike particles were gradually converted into spherical particles with the reaction continuing. It was found that the SERS enhancement depends on the size and aggregation of the silver particles, and the addition of $\mathrm{Cl}^{-}$ions generate much stronger SERS signals [14]. Promising SERS enhancements were obtained at composite nanoparticles [15]. The thin silver shell was electrodeposited on gold surface by controlling the applied potential and elec- trodeposition cycles. New SERS sensitive substrates allowed to use the single silver triangular nanoprisms [16].

The aim of this article is to present the unique analytical enhancement achieved for 4-aminothiophenol. The possibility for SAM participation was explained. We suppose the larger SERS enhancement capability of Ag$4 \mathrm{AMTPh}$ self-assembled monolayers (SAMs) provides the accessible surface area to the detected molecules. Besides, two silver nanoparticles adsorbed 4-AMTPhSAM have irregular polyhedron morphologies with distinct edges which could act as "hot sites" for surface plasmon resonance.

\section{Materials and methods}

All chemicals $\left(\mathrm{AgNO}_{3}, \mathrm{KCN}, \mathrm{KNO}_{3}\right.$, methanol and 4-aminothiophenol) were purchased from Alfa Aesar GmbH (Germany). They were of p.a. purity grade. The preparation of silver film was accomplished by Autolab PGSTAT302N (Utrecht, The Netherlands). The morphology and homogeneity of silver film was examined with scanning electron microscope (SEM, JOEL JSM-7001F, Japan). The operating voltage for the SEM was maintained at $10 \mathrm{kV}$ through the analysis. The identification of 4-aminothiophenol deposited on stainless steel and silver surface by SERS was performed by Raman spectrometer "Xplora" (Model BX41TF, HORIBA Jobin-Yvon, Japan) with wavelenght of $532 \mathrm{~nm}$. All the measuremnts were performed by conditions at $\sim 20^{\circ} \mathrm{C}$.

Electrochemical deposition of nanostructured Ag film was carried out in a conventional three-electrode cell employing Autolab PGSTAT302N. The counter electrode was a large-area platinum electrode. The reference electrode was an $\mathrm{Ag} / \mathrm{AgCl} / \mathrm{KCl}\left(3 \mathrm{~mol} / \mathrm{dm}^{3}\right)$ electrode. The working electrode was a stainless steel substrate of surface area $1 \mathrm{~cm}^{2}$. Electrochemical formation of $\mathrm{Ag}$ layer on stainless steel was performed from electrolyte containing gap $\mathrm{KNO}_{3}\left(0.1 \mathrm{~mol} / \mathrm{dm}^{3}\right)$, $\mathrm{KCN}\left(0.1 \mathrm{~mol} / \mathrm{dm}^{3}\right)$ and $\mathrm{AgNO}_{3}\left(0.01 \mathrm{~mol} / \mathrm{dm}^{3}\right)$. Silver layer was electrochemically prepared from working electrolyte by cyclic voltammetry method. The range of potentials was from $-0.700 \mathrm{~V}$ to $-1.55 \mathrm{~V}$. The scan rate was $0.1 \mathrm{~V} / \mathrm{s}$ and number of cycles was 15 . After preparation, the Ag films were rinsed with distilled water and stored in methanol to prevent oxidation. The stock and analytical solutions were freshly prepared with redistilled water.

\section{Results and disscusion}

The measurements at prepared silver nanostructured surface were aimed to determine mainly one surface function, that is analytical signal enhancement func- 

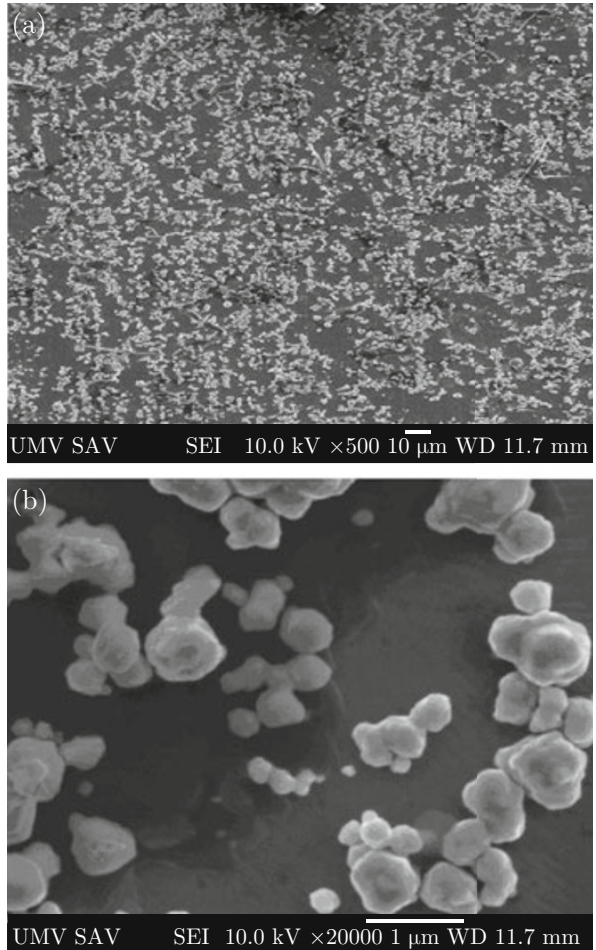

Fig. 1 SEM images of working electrode modified by $\mathrm{Ag}$ nanostructures. Magnification: (a) $500 \times$ and (b) $20000 \times$.

tion in Raman spectroscopy. Figure 1 shows SEM images of working electrode modified by $\mathrm{Ag}$ particles. The electrolytical deposition crystallized well and aggregated in polyhedron morphologies. The size of silver particles was up to $500 \mathrm{~nm}$. As a reference the stainless steel substrate was used in the experiment. The enhancement factor, $F_{e}$, was calculated according to the following formula,

$$
F_{e}=\frac{c_{\text {ref }}}{c_{\text {sample }}} \cdot \frac{I_{\text {sample }}}{I_{\text {ref }}}
$$

where $c_{\text {ref }}$ and $c_{\text {sample }}$ are the reference concentration and sample concentrations, respectively, and $I_{\text {ref }}$ is the signal intensity of the respective Raman peak.

The systematic SERS study of large ordered areas of organized silver oval nanoparticles (Fig. 1(b)) using 4-AMTPh as the probe molecule shows reproducible enhancement of the Raman signal. Enhancing factor was established of $1.2 \times 10^{3}$ for 4 -aminothiophenol concentration of $5 \times 10^{-4} \mathrm{~mol} / \mathrm{dm}^{3}$. The Raman shifts intensities for 4-aminothiophenol were shown in Table 1.

Concentration measurements confirmed detection limit for 4-AMTPh at level $10^{-16} \mathrm{~mol} / \mathrm{dm}^{3}$ (Fig. 2). The enhancement factor of $1.81 \times 10^{14}$ was achieved for a model analyte deposited on silver film. The enhancement of the peak located at $1163 \mathrm{~cm}^{-1}$ would be ascribed to the charge-transfer between the silver nanoparticles and the adsorbed molecules, which is usually dependent on the geometry of the molecules on the surface. We anticipate that the different behaviour for $5 \times 10^{-11} \mathrm{~mol} / \mathrm{dm}^{3} 4$-AMTPh may be due to the different "surface geometry" of the 4-AMTPh on small-sized $\mathrm{Ag}$ particles and dendrites (symmetry-breaking of the molecule).

According to Raman shift bands profile (Fig. 2) we can suppose participation of SAM at SERS signal enhancement. 4-AMTPh can form SAM and finally change dielectricum-silver layer sensitivity for adsorbate. In 4-AMTPh spectrum there absents S-H vibra-

Table 1 The Raman shift intensity values of working electrode modified with Ag nanostructured surfaces with different concentrations of 4-AMTPh

Concentration of 4-AMTPh $\left(\mathrm{mol} / \mathrm{dm}^{3}\right)$

Band of 4-AMTPh with Raman shift $\left(\mathrm{cm}^{-1}\right)$

\begin{tabular}{lccc}
\multicolumn{4}{c}{ Concentration of $4-\mathrm{AMTPh}\left(\mathrm{mol} / \mathrm{dm}^{3}\right)$} \\
\hline Reference & $5 \times 10^{-4}$ & $5 \times 10^{-13}$ & $5 \times 10^{-16}$ \\
\hline $5 \times 10^{-4}$ & \multicolumn{2}{c}{ Intensities/counts } \\
\hline \multicolumn{4}{c}{} \\
\hline 28.01 & 2154.44 & 911.23 & 565.88 \\
28.53 & 1948.98 & 794.55 & 559.80 \\
22.02 & 2883.38 & 1072.40 & 860.44 \\
27.91 & 4746.93 & 2228.62 & 1188.68 \\
34.41 & 1240.09 & 1081.50 & 745.95 \\
27.49 & 4122.47 & 2684.64 & 1771.06 \\
29.14 & 8357.87 & 5828.98 & 3210.69 \\
21.14 & 3777.08 & 5569.46 & 1049.42 \\
22.68 & 6659.23 & 3766.96 & 2628.33 \\
16.48 & 5667.95 & 3891.87 & 1482.98 \\
15.64 & 18769.10 & 9212.09 & 2824.97 \\
20.10 & 13332.08 & 3845.97 & 2271.26 \\
14.92 & 3932.26 & 2426.14 & 2900.65 \\
\hline
\end{tabular}




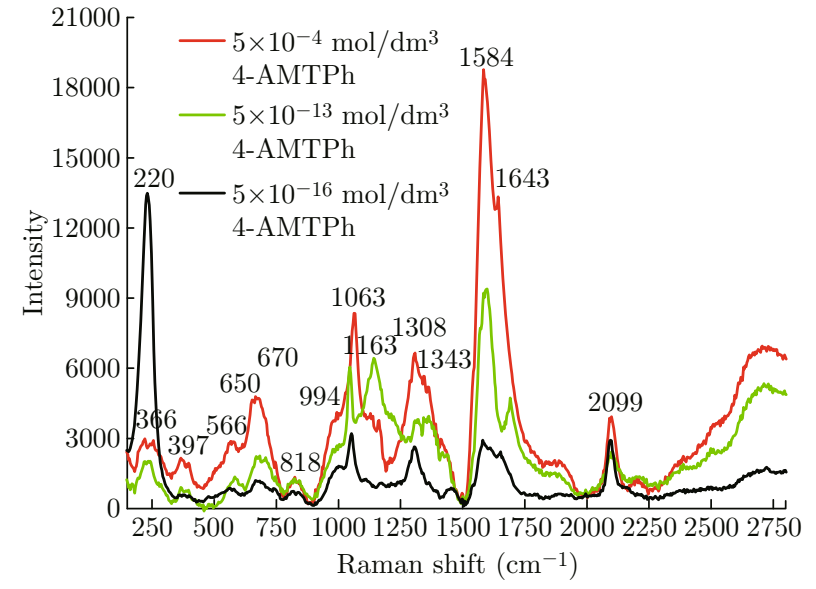

Fig. 2 SERS spectra of working electrode modified with $\mathrm{Ag}$ nanostructured surfaces in different concentrations of 4$\mathrm{AMTPh}$. The initial laser wavelength is $532 \mathrm{~nm}$.

tion at $2575 \mathrm{~cm}^{-1}$ from the molecule adsorbed on the metal surface. Another confirmation of SAM yield on SERS substrates is the high intensity of the C-S band at $650 \mathrm{~cm}^{-1}$ due to its proximity to the SERS active surface, also confirmed in references by Levin et al. [17] and Bryant et al. [18]. The Raman band at $200 \mathrm{~cm}^{-1}$ associated with Ag-S vibrations appeared, confirming the formation of SAM via the cleavage of the $\mathrm{S}-\mathrm{H}$ bond [19]. The results confirmed that SAM can be used to improve the SERS signal enhancement for SERSbased analytes and thus offering even higher sensitivity. Moreover, SERS enhancement can be systematically controlled based on several factors that include the structure of the SAM and the conditions under which SAM was formed.

As it is clear shown in Fig. 3, 4-AMTPh forms SAM where phenyl group forms "plant leaves field" and forms "hot spots" with silver nanoparticles. The values of an- alytical signal enhancement at different concentrations of 4-AMTPh can be found in Table 2. The formation of organic 4-AMTPh SAM layer on the silver nanoparticle film through their sulphur atoms is recently the well known and described phenomenon $[17,20,21]$.

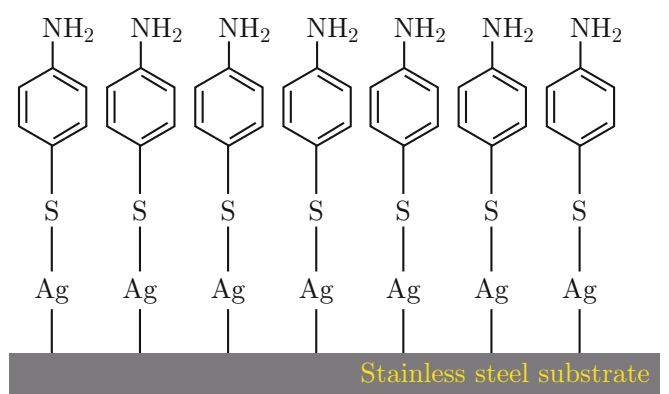

Fig. 3 Scheme of self-assembled monolayer of 4-AMTPh on a silver nanoparticle surface.

\section{Conclusion}

In this article we documented an unique signal enhancement for 4-aminothiophenol at Ag nanostructured surface using surface-enhanced Raman spectroscopy. It was confirmed that the testing analyte formed the selfassembled monolayers with silver substrate. This effect participated in signal enhancement of finally formed SERS active spots. The enhancing factor has been established at value of $1.81 \times 10^{14}$. Strong enhancement was probably influenced by both SERS and SAM participating mechanism.

\section{Acknowledgements}

This work was supported by the financial Grants VEGA 1/0211/12 and APPV-0280-11.

Table 2 The signal enhancement declared by Raman shift intensity values of working electrode modified with Ag nanostructured surfaces in different concentrations of 4-AMTPh

\begin{tabular}{|c|c|c|c|}
\hline \multirow{2}{*}{ Peak of 4-AMTPh with Raman shift $\left(\mathrm{cm}^{-1}\right)$} & \multicolumn{3}{|c|}{ Concentration of $4-\mathrm{AMTPh}\left(\mathrm{mol} / \mathrm{dm}^{3}\right)$} \\
\hline & $5 \times 10^{-4}$ & $5 \times 10^{-13}$ & $5 \times 10^{-16}$ \\
\hline 365.78 & $0.77 \times 10^{2}$ & $0.33 \times 10^{11}$ & $0.20 \times 10^{14}$ \\
\hline 396.84 & $0.68 \times 10^{2}$ & $2.79 \times 10^{11}$ & $0.20 \times 10^{14}$ \\
\hline 565.98 & $1.30 \times 10^{2}$ & $0.49 \times 10^{11}$ & $0.39 \times 10^{14}$ \\
\hline 669.53 & $1.70 \times 10^{2}$ & $0.80 \times 10^{11}$ & $0.43 \times 10^{14}$ \\
\hline 817.95 & $0.36 \times 10^{2}$ & $0.31 \times 10^{11}$ & $0.22 \times 10^{14}$ \\
\hline 993.98 & $1.50 \times 10^{2}$ & $0.98 \times 10^{11}$ & $0.64 \times 10^{14}$ \\
\hline 1063.02 & $2.87 \times 10^{2}$ & $2.00 \times 10^{11}$ & $1.10 \times 10^{14}$ \\
\hline 1163.12 & $1.79 \times 10^{2}$ & $2.64 \times 10^{11}$ & $0.50 \times 10^{14}$ \\
\hline 1308.09 & $2.94 \times 10^{2}$ & $1.66 \times 10^{11}$ & $1.16 \times 10^{14}$ \\
\hline 1342.60 & $3.44 \times 10^{2}$ & $2.36 \times 10^{11}$ & $0.90 \times 10^{14}$ \\
\hline 1584.22 & $12.00 \times 10^{2}$ & $5.89 \times 10^{11}$ & $1.81 \times 10^{14}$ \\
\hline 1642.90 & $6.63 \times 10^{2}$ & $1.91 \times 10^{11}$ & $1.13 \times 10^{14}$ \\
\hline 2098.52 & $2.64 \times 10^{2}$ & $1.63 \times 10^{11}$ & $1.94 \times 10^{14}$ \\
\hline
\end{tabular}




\section{References}

[1] B. Reents, G. Lacconi and W. Plieth, J. Electroanal. Chem. 376, 185 (1994). http://dx.doi.org/10.1016/ 0022-0728 (94) 03615-2

[2] F. Fathi, F. Lagugn, Labarthet, D. B. Pedersen and H. B. Kraatz, Analyst 137, 4448 (2012). http://dx.doi. org/10.1039/c2an35641d

[3] M. Potara, M. Baia, C. Farcau and S. Astilean, Nanotechnology 23, 055501 (2012). http://dx.doi.org/ 10.1088/0957-4484/23/5/055501

[4] M. Moskovits, Rev. Mod. Phys. 57, 783 (1985). http://dx.doi.org/10.1103/RevModPhys.57.783

[5] A. Otto, J. Raman Spectrosc. 22, 743 (1991). http:// dx.doi.org/10.1002/jrs.1250221204

[6] U. Kreibig and M. Vollmer, Properties of Metal Clusters, Springer Series in Metal Science, 25, 1995.

[7] M. J. Mulvihill, X. Y. Ling, J. Henzie and P. Yang, J. Am. Chem. Soc. 132, 268 (2010). http://dx.doi. org/10.1021/ja906954f

[8] P. M. Shirage, D. D. Shivagan, L. A. Ekal, N. V. Desai, S. B. Mane and S. H. Pawar, Appl. Surf. Sci. 182, 403 (2001). http://dx.doi.org/10.1016/ S0169-4332(01) 00459-7

[9] R. K. Chang and T. E. Furtak (Eds.) Surface Enhanced Raman Scattering, Plenum, New York, 1982.

[10] W. Plieth, H. Dietz, A. Anders, G. Sandmann, A. Meixner, M. Weber and H. Kneppe, Surf. Sci. 597, 119 (2005). http://dx.doi.org/10.1016/j. susc. 2004.02.042

[11] R. Kodiyath, J. Wang, Z. A. Combs, S. Chang, M. K. Gupta, K. D. Anderson, R. J. C. Brown and V. V.
Tsukruk, Small 7, 3452 (2011). http://dx.doi.org/ 10.1002/smll. 201101936

[12] E. S. Papazoglou, S. Babu, S. Mohapatra, D. R. Hansberry and C. Patel, Nano-Micro Lett. 2, 74 (2010). http://dx.doi.org/10.5101/nml.v2i2. p74-82

[13] K. G. Stamplecoskie, J. C. Scaiano, V. S. Tiwari and H. Anis, J. Phys. Chem. C 115, 1403 (2011). http:// dx.doi.org/10.1021/jp106666t

[14] X. Li, J. Zhang, W. Xu, H. Jia, X. Wang, B. Yang, B. Zhao, B. Li and Y. Ozaki, Langmuir 19, 4285 (2003). http://dx.doi.org/10.1021/la0341815

[15] J. Deng, J. Du, Y. Wang, Y. Tu and J. Di, Electrochem. Commun. 13, 1517 (2011). http://dx.doi. org/10.1016/j.elecom. 2011.10.010

[16] L. J. Sherry, R. Jin, Ch. A. Mirkin, G. C. Schatz and R. P. Van Duyne, Nano Lett. 6, 2060 (2006). http:// dx.doi.org/10.1021/nl061286u

[17] C. S. Levin, B. G. Janesko, R. Bardhan, G. E. Scuseria, J. D. Hartgerink and N. J. Halas, Nano Lett. 6, 2617 (2006). http://dx.doi.org/10.1021/nl062283k

[18] M. A. Bryant and J. E. Pemberton, J. Am. Chem. Soc. 113, 8284 (1991). http://dx.doi.org/10.1021/ ja00022a014

[19] Ch. K. Klutse, A. Mayer, J. Wittkamper and B. M. Cullum, J. Nanotechnol. Article ID 319038 (2012). http://dx.doi.org/10.2116/analsci.26.957

[20] Y. Yhou, J. Yhi, J. Yhao and M. Xu, Anal. Sci. 26, 957 (2010). http://dx.doi.org/10.2116/ analsci.26.957

[21] Q. Zhou, X. X. Zhang, F. Z. Pang, X. W. Li, J. W. Zheng and T. H. Lu, Acta Chim. Sinica 63, 1561 (2005). 